\title{
Tail behaviour of the area under a random process, with applications to queueing systems, insurance and percolations
}

\author{
Rafał Kulik • Zbigniew Palmowski
}

Received: 10 May 2011 / Revised: 10 May 2011 / Published online: 9 July 2011

(C) The Author(s) 2011. This article is published with open access at Springerlink.com

\begin{abstract}
The areas under the workload process and under the queueing process in a single-server queue over the busy period have many applications not only in queueing theory but also in risk theory or percolation theory. We focus here on the tail behaviour of distribution of these two integrals. We present various open problems and conjectures, which are supported by partial results for some special cases.
\end{abstract}

Keywords Queueing process · Risk process · Workload · Area · Busy period

Mathematics Subject Classification (2000) 60G07

\section{Introduction}

In the past two decades an enormous amount of work on asymptotics for queueing systems has been done. The tail behaviour of steady-state queueing process $\{Q(t), t \geq 0\}$, the workload $\{W(t), t \geq 0\}$ or the busy period $\tau$ in standard systems has been well understood in both light- and heavy-tailed case. Surprisingly, however, very little is known on tail behaviour of integral functionals of the form

$$
I_{f}(T):=\int_{0}^{T} f(X(u)) d u,
$$

R. Kulik

Department of Mathematics and Statistics, University of Ottawa, 585 King Edward Av., K1N 6N5 Ottawa, ON, Canada

e-mail:rkulik@uottawa.ca

Z. Palmowski (凶)

Mathematical Institute, Wrocław University, Pl. Grunwaldzki 2/4, 50-384 Wrocław, Poland

e-mail: zpalma@math.uni.wroc.pl 
where $\{X(t), t \geq 0\}$ is a stochastic process (typically, $X=Q$ or $X=W$ ), $f$ is a deterministic function and $T$ is either $\tau$ or deterministic (finite or infinite). Such integrals appear naturally in analysis of ATM. The reader is referred to references given in [9] and [21]. Recently, in [2], the authors connected mean bit rate in time varying $M / M / 1$ queue with moments of the integral $\int_{0}^{\tau} Q(u) d u$ in a corresponding standard $M / M / 1$ system.

However, applications of such integrals go beyond queueing systems. Let $\{S(t), t \geq 0\}$ be a standard risk process. Integrals $\int_{0}^{T} 1_{\{S(u)<0\}} S(u) d u$, where $T$ is deterministic (i.e. integrated negative part of the risk process), are suggested in [8] as possible risk measures. Further extensions are given in a multivariate setting. Furthermore, as in [16], integrals $\int_{0}^{\tau} Q(u) d u$ in Geo/Geo/1 queues and (as a limit) in $M / M / 1$ systems have particular interpretation in compact percolations. Last but not least, if $X$ is Lévy process, integrals $\int_{0}^{\infty} \exp (-X(u)) d u$ have applications in financial mathematics; see [24]. Other applications are coming from actuarial science, where very often regulated processes are considered and integral functionals from a regulation random mechanism are investigated.

\section{Subexponential asymptotics}

Consider a stable $G I / G I / 1$ queue. Denote by $\left\{T, T_{i}, i \geq 0\right\}$ and $\left\{S, S_{i}, i \geq 0\right\}$ two stationary i.i.d. and mutually independent sequences of interarrival and service times, respectively. Let $\lambda_{T}=1 / \mathrm{E}[T], \lambda_{S}=1 / \mathrm{E}[S]$ and $\rho=\lambda_{T} / \lambda_{S}$. Let $\{Q(t), t \geq 0\}$ be a stationary queueing process and $\tau=\inf \{t \geq 0: Q(t)=0\}$ the corresponding busy period. We shall assume that the distribution $F$ of service time $S$ is subexponential (denoted as $F \in \mathcal{S}$ ). The distribution $G$ is subexponential when

$$
\lim _{x \rightarrow \infty} \overline{G^{* 2}}(x) / \bar{G}(x)=2,
$$

where $G^{* 2}$ is the convolution of $G$ with itself, and $\bar{G}$ denotes the tail distribution given by $\bar{G}(x)=1-G(x)$.

Heuristically, the large area $\int_{0}^{\tau} Q(u) d u$ is realized by a customer with large service time $S_{0}$, say, who arrives at the very beginning of the busy period and blocks the server. During that time $S_{0}$, according to the Law of Large Numbers (LLN), approximately $\lambda_{T} S_{0}$ customers arrive, and the queue length process increases linearly. Hence the area under the queueing process before reaching maximum is asymptotically equivalent to the area of a triangle: $\frac{1}{2 \lambda_{T}} \bar{Q}(\tau)^{2}$, where $\bar{Q}(\tau)$ is maximum of the queue length process over the busy period (see Fig. 1). After passing the maximum, the queue length process behaves according to the LLN, decreasing almost linearly to 0 with the slope $\lambda_{S}-\lambda_{T}$. Thus, the area under the queueing process after reaching the maximum is equivalent to $\frac{1}{2\left(\lambda_{S}-\lambda_{T}\right)} \bar{Q}(\tau)^{2}$, and $\bar{Q}(\tau)$ is equivalent to $\rho\left(\lambda_{T}-\lambda_{S}\right) \tau$. This heuristic leads to the following conjecture.

Conjecture 2.1 If $F \in \mathcal{S}$, then

$$
P\left(\int_{0}^{\tau} Q(u) d u>x\right) \sim P\left(\tau>\sqrt{\frac{2 x}{\rho\left(\lambda_{S}-\lambda_{T}\right)}}\right) .
$$


Fig. 1 A typical behaviour of a heavy-tailed queueing process

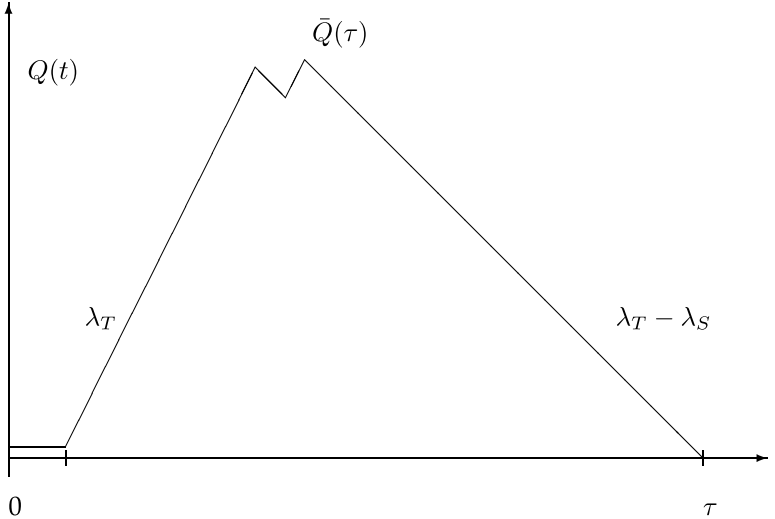

Similarly, for the workload process the heuristic is as follows. The most likely way for the area to be large is that one early big service time occurs and apart from this, everything in the cycle develops normally. Using LLN and ignoring random fluctuations, this leads to the conclusion that the workload goes to zero with negative rate $-(1-\rho)$. Thus the area exceeds level $x$ iff the area of the triangle with the sides $\tau(1-\rho)$ and $\tau$ is greater than $x$, hence when

$$
\frac{1}{2} \tau^{2}(1-\rho)>x
$$

which suggests the following conjecture.

Conjecture 2.2 If $\bar{F} \in \mathcal{S}$, then

$$
P\left(\int_{0}^{\tau} W(u) d u>x\right) \sim P\left(\tau>\sqrt{\frac{2 x}{1-\rho}}\right) .
$$

Statements (2) and (3) were proven in [21] and [9], respectively, under regularly varying assumption of the service time, that is $\bar{F}(x)=x^{-\alpha} L(x)$, where $\alpha>1$, and $L$ is slowly varying at infinity. Furthermore, in [21] one needs additionally that

$$
\lim _{t \rightarrow \infty} \frac{t^{1+\varsigma} P(T>t)}{\bar{F}(t)}=0
$$

with $\varsigma>0$.

The tail behaviour of the busy period $\tau$ can be identified in terms of $\bar{F}$ for a large subclass of $\mathcal{S}$ :

$$
P(\tau>x) \sim \frac{1}{1-\rho} \bar{F}((1-\rho) x),
$$

see $[5,15]$ and [28]. In particular, using (5) in the regularly varying case, together with (2) and (3), yields exact asymptotics for area under queueing process and workload, respectively (see [21] and [9]). 


\section{Light-tailed asymptotics}

As in Sect. 2, we consider a stable $G I / G I / 1$ queue. Here, we assume that the service time $S$ is light-tailed, that is there exists $\theta>0$ such that $\mathrm{E}[\exp (\theta S)]<\infty$.

Under the above assumptions for the queueing process, we have the following open problem:

Open Problem 3.1 Find exact asymptotics of

$$
P\left(\int_{0}^{\tau} Q(u) d u>x\right) .
$$

We suppose that

$$
P\left(\int_{0}^{\tau} Q(u) d u>x\right) \sim C x^{-1 / 4} \exp (-\psi \sqrt{x})
$$

for some constants $\psi$ and $C$.

We suggest the above asymptotics believing that it should be the same as for the $M / M / 1$ queue, which was found in [13] under two conjectures on p. 391 and is in the following form:

$$
P\left(\int_{0}^{\tau} Q(u) d u>x\right) \sim \frac{1-\rho}{\rho \sqrt{2 \pi \psi}} x^{-1 / 4} \exp (-\psi \sqrt{x}),
$$

where

$$
\psi=2 \sqrt{-2(1-\rho)+(1+\rho) \log \rho} .
$$

In the proof the authors used the Laplace transform method. We are not aware of any probabilistic proof of this result, and we do not know if Conjectures 1 and 2 in [13] hold true.

Unfortunately, we have not managed to produce any heuristic for this result either. The idea of the piecewise linear most likely trajectory seems to produce the wrong expression. In particular, define the new probability measure $\tilde{P}$ by

$$
\frac{d \tilde{P}_{\mid \mathcal{F}_{n}}}{d P_{\mid \mathcal{F}_{n}}}=e^{\gamma \sum_{i=1}^{n}\left(S_{i}-T_{i-1}\right)}
$$

where $\mathcal{F}_{n}=\sigma\left(T_{1}, S_{1}, \ldots, T_{n}, S_{n}\right)$, and $\gamma$ solves the equation $\mathrm{E}[\exp (\gamma(T-S))]=1$. Let $\tilde{\rho}=\frac{\tilde{\mathrm{E}}[T]}{\tilde{\mathrm{E}}[S]}>1$. Consider the most likely path coming from large deviation theory for large cycle maxima, that is the trajectory that develops along the line with the slope $\tilde{\rho}-1$ and after reaching its maximum behaves 'normally', that is, goes to zero linearly with negative rate $-(1-\rho)$. This trajectory produces wrong asymptotics for the $M / M / 1$ queue since by Kyprianou [18] we have then:

$$
P\left(\int_{0}^{\tau} Q(u) d u>x\right) \sim P\left(\tau>\sqrt{2 \frac{1+\rho}{1-\rho} x}\right) \sim C x^{-3 / 4} e^{-\gamma \sqrt{2 \frac{1+\rho}{1-\rho}} \sqrt{x}}
$$


for some constant $C$ and $\gamma=(1-\sqrt{\rho})^{2} \mu$. The explanation might come from papers $[11,25,26]$, where the optimal trajectories in a sense of large deviation theory for the mean value of a reflected random walk $W_{n}$ are considered. The relationship between area under the queue length and sum of $W_{n}$ is clear when observing the queue length process at arrival and departure epochs. These papers prove that for deterministic terminal times, the optimal path, though still concave in general, might not be piecewise linear. In fact, these papers show that the optimal trajectory $q(t)$ of the queueing process for the large value of the area on the cycle should solve the following equation:

$$
\nabla I\left(\frac{d}{d t} q(t)\right)=\lambda^{*}(\tau-t)
$$

where $\lambda^{*}>0$, and $I$ is a rate function for the increment process. The proof of Problem 3.1 would then require an extension of this result to the case of a random terminal time being the end of busy period. Apart from it, solving (8) explicitly seems to be a difficult task and transferring it into finding the asymptotic tail distribution of $\tau$ and the integral even more cumbersome. This problem shows also the need of simulations of the area $\int_{0}^{\tau} Q(u) d u$ to check if (8) indeed produces non-piecewise-linear optimal path. In general, we are not aware of any simulations results concerning area under a random process.

One can pose a similar open problem for $P\left(\int_{0}^{\tau} W(u) d u>x\right)$.

\section{Extensions}

\subsection{Transient case}

Let us consider the $M / M / 1$ queue with $\rho=1$. In [16] the author established the following asymptotics for the critical case $\rho=1$ :

$$
P\left(\int_{0}^{\tau} Q(u) d u>x\right) \sim \frac{3^{1 / 3}}{\Gamma(1 / 3) \psi_{0} \psi_{1}} x^{-1 / 3},
$$

where $\psi_{0}$ and $\psi_{1}$ are explicit constants.

Open Problem 4.1 Consider a transient $G I / G I / 1$ queue such that $\bar{F}(x)$ is regularly varying. What the tail asymptotics of $\int_{0}^{\tau} Q(u) d u$ and $\int_{0}^{\tau} W(u) d u$ ?

As mentioned above in the Introduction, these type of questions should have connections with heavy-tailed critical percolations.

\subsection{Utility functions, discounting}

Consider again a stable $G I / G I / 1$ queue. Assume that $f$ is a deterministic function. We are interested in the tail behaviour of $\int_{0}^{\tau} f(W(u)) d u$. In an insurance context, $f$ may play the role of the utility function. Of course, one can formulate the corresponding problem for the queueing process, where $f$ gives the costs of maintaining the system. 
The heuristic given in the subexponential case in Sect. 2 suggests that studying the asymptotics of the latter integral is equivalent to studying the behaviour of

$$
P\left(\int_{0}^{\tau} f((\tau-u)(1-\rho)) d u>x\right)
$$

when $x$ becomes large. For example, if one considers $f(x)=x^{k}, k \geq 0$, it leads to the following conjecture.

Conjecture 4.1 Assume that $\bar{F} \in \mathcal{S}$ and $f(x)=x^{k}$. Then,

$$
P\left(\int_{0}^{\tau} f(W(u)) d u>x\right) \sim P\left(\tau>\sqrt[k+1]{\frac{(k+1) x}{(1-\rho)^{k}}}\right) .
$$

However, it is not clear for us what should be expected for a very general function $f$.

Now, assume that $\theta>0$ and consider the process $X(t)=\exp (-\theta t) f(W(t))$, $t \geq 0$. Similarly, the corresponding integral can be interpreted in an insurance context: $\exp (-\theta t)$ is the discounting factor, and $f$ is the utility function.

The subexponential heuristic leads us again to

$$
P\left(\int_{0}^{\tau} \exp (-\theta u) f((\tau-u)(1-\rho)) d u>x\right) .
$$

For further heuristic, consider $f(x)=x^{k}$. Then

$$
\begin{aligned}
& \int_{0}^{\tau} \exp (-\theta u) f((\tau-u)(1-\rho)) d u=(1-\rho)^{k} \int_{0}^{\tau} \exp (-\theta u)(\tau-u)^{k} d u \\
& \quad=(1-\rho)^{k} \exp (-\theta \tau) \int_{0}^{\tau} \exp (\theta s) s^{k} d s \\
& \quad=(1-\rho)^{k} \exp (-\theta \tau)\left[\frac{1}{\theta} \exp (\theta \tau) \tau^{k}-\frac{k}{\theta} \int_{0}^{\tau} \exp (\theta s) s^{k-1} d s\right] .
\end{aligned}
$$

Integrating further, by parts, the last integral, we see that the leading term will be $O\left(\exp (\theta \tau) \tau^{k-1}\right)$. This leads to the following conjecture.

Conjecture 4.2 Assume that $F \in \mathcal{S}$ and $f(x)=x^{k}$. Then,

$$
P\left(\int_{0}^{\tau} \exp (-\theta u) f(W(u)) d u>x\right) \sim P\left(\tau>\sqrt[k]{\frac{\theta x}{(1-\rho)^{k}}}\right) .
$$

This conjecture should be compared with Conjecture 4.1, showing how discounting leads to the change in the asymptotic behaviour.

Let us consider now a light-tailed case. The large area for the integral $\int_{0}^{\tau} W(u) d u$ is strictly connected to a large $\bar{W}(\tau)$. It does not seem to be the case if one considers $\int_{0}^{\tau} \exp (-\theta u) f(W(u)) d u$, i.e. large values of $W(u)$ may be killed by the discount factor. 
Open Problem 4.2 Under the conditions of Open Problem 3.1, find the asymptotics for

$$
P\left(\int_{0}^{\tau} \exp (-\theta u) f(W(u)) d u>x\right)
$$

\subsection{Finite horizon}

All the conjectures and open problems above may be formulated in case of $\int_{0}^{T}$, where $T$ is finite and deterministic. In particular, if $S(t)=v+c t-\sum_{i=1}^{N(t)} S_{i}, t \geq 0$, is the classical risk insurance process, then moments of

$$
\int_{0}^{T} S(u) 1_{\{S(u)<0\}} d u
$$

are proposed in [8] as particular risk measures. In that paper the authors established the asymptotics for the expected value of the integral when $T$ is fixed and the initial capital $v$ becomes large. Clearly, this problem can be re-formulated for the dual workload process. Furthermore, all the above-mentioned extensions (discounting, utility functions) may be considered.

\subsection{Multivariate case}

As in Sect. 4.3, let us consider insurance context. We assume that a company has two lines of business and customers arrive according to a renewal process $N(t)$, $t \geq 0$, with generic interarrival time $T$. The $i$ th customer has a claim $\left(S_{i, 1}, S_{i, 2}\right)$. For example, a car accident may cause a claim for driving and liability insurance (see for example $[4,8]$ and references therein). If two lines of business are considered separately, we are interested in the tail behaviour of

$$
\left(\int_{0}^{T} S_{1}(u) 1_{\left\{S_{1}(u)<0\right\}} d u, \int_{0}^{T} S_{2}(u) 1_{\left\{S_{2}(u)<0\right\}} d u\right),
$$

where $S_{1}$ and $S_{2}$ are risk process associated with the corresponding lines of business. One can consider the tail behaviour when $T$ is fixed and a vector of initial capital $\left(x_{1}, x_{2}\right)$ becomes large (in a particular sense, usually dependence is linear, that is $x_{1}=a x_{2}$ for some fixed $a>0$ ).

In queueing theory one can consider, for instance, parallel queues, where customers are coming into the system according to renewal process $N(t)$ and each service time (we assume here that all of them are i.i.d.) is proportionally divided between two servers (see for example [22] and [23]). In this case, $S_{i, 1}=b S_{i, 2}$ for some fixed $b$. Let $W_{i}(t)$ and $Q_{i}(t)$ be the workload and queueing process on the $i$ th server $(i=1,2)$. The busy period $\tau$ is understood here as the minimum of the busy periods on both servers. We could analyze the tail behaviour of the following "tail" probability:

$$
P\left(\int_{0}^{\tau} W_{1}(u) d u>x \text { and } \int_{0}^{\tau} W_{2}(u) d u>a x\right) \text { as } x \rightarrow \infty
$$


under the assumption that the distribution $F$ of $S_{i, 1}$ is subexponential or light-tailed. Similar considerations could be analyzed for the bivariate occupation process.

\subsection{Regulated processes}

Apart from the original process $X(t)$ in (1), one can consider the regulated process

$$
U(t)=X(t)-L(t)
$$

where $L(t)$ is a left-continuous, increasing process, adapted with respect to the natural filtration of $X$. For example, if $X$ is a spectrally negative Lévy risk process, then $L(t)$ might be cumulative dividends payment up to time $t$ payed according to some strategy. The most often used strategy is the so-called barrier strategy in which all surpluses above a given level $a$ are transferred (possibly subject to a discount rate) to a beneficiary. In this case, $L(t)=a \vee \sup _{s \leq t} X(s)-a$, and for given utility function, we are interested in the following random variable:

$$
I_{f}(\tau)=\int_{0}^{\tau} e^{-\theta u} f(L(u)) d u
$$

(possibly with $\theta=0$ ), where $\tau=\inf \{t \geq 0: U(t)<0\}$ is a ruin time.

We can also analyze discounted cumulative dividends payed up to ruin time:

$$
\int_{0}^{\tau} e^{-\theta u} d L(u)
$$

which is equivalent to finding the tail asymptotics of the integral

$$
\int_{0}^{\infty} e^{-\theta Z_{1}(t)} \mathbf{1}_{\left\{\Delta Z_{2}(u)<a\right\}} d u,
$$

where $\left(Z_{1}(t), Z_{2}(t)\right)$ is a bivariate subordinator, and $\Delta Z_{2}(u)=Z_{2}(u)-Z_{2}(u-)$ is the jump size of $Z_{2}$ at time $u$ (see [1,3,20,27] for details and other references). In the case $a=\infty$, we end up with an integral from the exponential function of a Lévy process; see for example $[6,7,10,24]$ for this kind of functional. Note that in particular $I=\int_{0}^{\infty} e^{-\theta Z_{1}(t)} d u$ solves the following equation: $I \stackrel{\mathcal{D}}{=} \int_{0}^{\tau} e^{-\theta Z_{1}(t)} d u+$ $e^{-\theta Z_{1}(\tau)} I$, and analyzing $I$ is strongly related then with properties of $e^{-\theta Z_{1}(\tau)}$ (see for example $[12,17])$.

With this problem there is also a related integral with respect to the general Lévy process:

$$
\int_{0}^{T} Y(u) d X(u)
$$

for appropriate predictable integrand $Y$ and fixed $T$ (see [14] for the multivariate regularly varying setting).

In queueing systems with single server and finite capacity $a, L(t)$ corresponds then to cumulative loss of work. If only a proportion of information is lost, then we 
can consider, for instance, $L(t)=c \mathbf{1}_{\{U(t)>a\}}$ for some $c>0$. In this case formally the so-called refracted process $U$ solves the following stochastic equation:

$$
d U(t)=d X(t)-c \mathbf{1}_{\{U(t)>a\}} d t
$$

see [19]. In this case we can also try to find an asymptotic tail of (10) and (11).

Acknowledgements The authors thank Sean P. Meyn for inspiring discussions and suggestion of optimal path (8). This work is partially supported by the Ministry of Science and Higher Education of Poland under the grants N N201 394137 (2009-2011) and N N201 525638 (2010-2011).

Open Access This article is distributed under the terms of the Creative Commons Attribution Noncommercial License which permits any noncommercial use, distribution, and reproduction in any medium, provided the original author(s) and source are credited.

\section{References}

1. Albrecher, H., Thonhauser, S.: Optimality results for dividend problems in insurance. RACSAM Rev. R. Acad. Cien. Ser. A. Mat. 103(2), 295-320 (2009)

2. Antunes, N., Fricker, C., Guillemin, F., Robert, P.: Perturbation analysis of the area swept under the queue length process of a variable $M / M / 1$ queue. Performance (2005)

3. Avram, F., Palmowski, Z., Pistorius, M.: On the optimal dividend problem for a spectrally negative Lévy process. Ann. Appl. Probab. 17, 156-180 (2007)

4. Avram, F., Palmowski, Z., Pistorius, M.: Exit problem of a two-dimensional risk process from a cone: exact and asymptotic results. Ann. Appl. Probab. 18(6), 2421-2449 (2008)

5. Baltrunas, A., Daley, D.J., Klüppelberg, C.: Tail behaviour of the busy period of a $G I / G I / 1$ queue with subexponential service times. Stoch. Process. Appl. 111, 237-258 (2004)

6. Bertoin, J., Yor, M.: On the entire moments of self-similar Markov processes and exponential functionals of Lévy processes. Ann. Fac. Sci. Toulouse Math. (6) 11(1), 33-45 (2002)

7. Bertoin, J., Biane, P., Yor, M.: Poissonian exponential functionals, $q$-series, $q$-integrals, and the moment problem for log-normal distributions. In: Seminar on Stochastic Analysis, Random Fields and Applications IV. Progr. Probab., vol. 58, pp. 45-56. Birkhäuser, Basel (2004)

8. Biard, R., Loisel, S., Maccib, C., Veraverbeke, N.: Asymptotic behavior of the finite-time expected time-integrated negative part of some risk processes and optimal reserve allocation. J. Math. Anal. Appl. 367(2), 535-549 (2010)

9. Borovkov, A.A., Boxma, O.J., Palmowski, Z.: On the integral of the workload process of the single server queue. J. Appl. Probab. 40, 200-225 (2003)

10. Carmona, P., Petit, F., Yor, M.: On the distribution and asymptotic results for exponential functionals of Lévy processes. In: Exponential Functionals and Principal Values Related to Brownian Motion. Bibl. Rev. Mat. Iberoamericana, pp. 73-130 (1997)

11. Duffy, K.R., Meyn, S.P.: Most likely paths to error when estimating the mean of a reflected random walk. Perform. Eval. 67(12), 1290-1303 (2010)

12. Goldie, C.M.: Implicit renewal theory and tails of solutions of random equations. Ann. Appl. Probab. 1(1), 126-166 (1991)

13. Guillemin, F., Pinchon, D.: On the area swept under the occupation process of an $M / M / 1$ queue in a busy period. Queueing Syst. 29, 383-398 (1998)

14. Hult, H., Linskog, F.: Extremal behavior of stochastic integrals driven by regularly varying Lévy processes. Ann. Probab. 35, 309-339 (2007)

15. Jelenković, P.R., Momčilović, P.: Large deviations of square root insensitive random sums. Math. Oper. Res. 29, 398-406 (2004)

16. Kearney, M.J.: On a random area variable arising in discrete-time queues and compact directed percolation. J. Phys. A, Math. Gen. 37, 8421-8431 (2004)

17. Kesten, H.: Random difference equations and renewal theory for products of random matrices. Acta Math. 131, 207-248 (1973) 
18. Kyprianou, E.K.: On the quasi-stationary distribution of the virtual waiting time in queues with Poisson arrivals. J. Appl. Probab. 8, 494-507 (1971)

19. Kyprianou, A., Loeffen, R.: Refracted Lévy processes. Ann. Inst. H. Poincaré Probab. Stat. 46, 24-44 (2010)

20. Kyprianou, A., Palmowski, Z.: Distributional study of De Finetti's dividend problem for a general Lévy insurance risk process. J. Appl. Probab. 44(2), 428-443 (2007)

21. Kulik, R., Palmowski, Z.: Tail behaviour of the area under queue length process of a single server queue with regularly varying service times. Queueing Syst. 50, 299-323 (2005)

22. Lieshout, P., Mandjes, M.: Asymptotic analysis of Levy-driven tandem queues. Queueing Syst. 60, 203-226 (2008)

23. Lieshout, P., Mandjes, M.: Brownian tandem queues. Math. Methods Oper. Res. 66, 275-298 (2007)

24. Maulik, K., Zwart, B.: Tail asymptotics for exponential functionals of Lévy processes. Stoch. Process. Appl. 116, 156-177 (2006)

25. Meyn, S.P.: Large deviation asymptotics and control variates for simulating large functions. Ann. Appl. Probab. 16(1), 310-339 (2006)

26. Meyn, S.P.: Control Techniques for Complex Networks. Cambridge University Press, Cambridge (2007)

27. Schmidli, H.: Stochastic Control in Insurance. Springer, New York (2008)

28. Zwart, A.P.: Tail asymptotics for the busy period in the $G I / G / 1$ queue. Math. Oper. Res. 26, 485-493 (2001) 